\title{
Integración arquitectónica de colectores solares térmicos cerámicos para clima mediterráneo
}

\section{Architectural integration of energy solar collectors made with ceramic materials and suitable for the Mediterranean climate}

$\underline{\text { J. Roviras }}^{(*)}$, V. Sarrablo ${ }^{(*)}$, M. M. Casanovas ${ }^{(*)}$, J. Armengou ${ }^{(*)}$

\section{RESUMEN}

El trabajo aquí presentado se orienta a demostrar la viabilidad técnica, arquitectónica y energética de colectores solares térmicos realizados con materiales cerámicos y adecuados al clima mediterráneo para la producción de agua caliente sanitaria (ACS) y de calefacción en edificios. Se propone el diseño de una envolvente cerámica formada por paneles captadores y paneles no captadores, que forman parte de un mismo sistema constructivo que es capaz de dar respuesta a los requerimientos básicos de un cerramiento exterior y de captar la energía solar. La cerámica consigue reducir considerablemente el coste final del sistema captador y ofrece al nuevo sistema una gran variedad de juego compositivo y cromático dado que, con un menor rendimiento frente al de un colector convencional metálico, puede ocupar toda la superficie de fachada y obtener un elevado grado de integración arquitectónica. También se ha definido una herramienta que permite evaluar el sistema de captación solar térmica cerámico desde un punto de vista multicriterio, económico, ambiental y social, para compararlo con los sistemas de captación solar térmica comerciales bajo distintas condiciones climáticas y de demanda.

Palabras clave: colector solar integrado; colector solar cerámico; integración arquitectónica; materiales cerámicos sostenibles; análisis multicriterio.

\section{ABSTRACT}

The work presented here aims to demonstrate the technical, architectural and energy viability of solar thermal collectors made with ceramic materials and the Mediterranean climate suitable for the production of domestic hot water (DHW) and for heating systems in buildings. The design of a ceramic shell formed by panels collectors and panels no sensors, which are part of the same building system that is capable of responding to the basic requirements of a building envelope and capture solar energy is proposed. Ceramics considerably reduced the final cost of the sensor system and offers the new system a variety of compositional and chromatic since, with reduced performance compared to a conventional metallic collector, can occupy the entire surface of front and get a high degree of architectural integration. A tool for assessing the new ceramic solar collector has been defined from a multi-criteria perspective: economic, environmental and social. The tool enables the comparison of the ceramic solar collector with solar collectors on the market under different climatic and demand conditions.

Keywords: building integrated solar collector; ceramic solar collectors; architectural integration; sustainable ceramic materials; multi-criteria analysis.

(*) Universitat Internacional de Catalunya. Barcelona (España)

Persona de contacto/Corresponding author: jroviras@uic.es (J. Roviras)

Cómo citar este artículo/Citation: Roviras, J., Sarrablo, V., Casanovas, M. M., Armengou, J. (2016). Integración arquitectónica de colectores solares térmicos cerámicos para clima mediterráneo. Informes de la Construcción, 68(544): e163, doi: http://dx.doi. org/10.3989/ic.16.001.m15.

Copyright: (C) 2016 CSIC. Licencia / License: Salvo indicación contraria, todos los contenidos de la edición electrónica de Informes de la Construcción se distribuyen bajo una licencia de uso y distribución Creative Commons Attribution License (CC BY) Spain 3.o. 


\section{INTRODUCCIÓN}

La importancia del consumo de energía en relación con los problemas del medio ambiente y el agotamiento de los recursos naturales ha incrementado el fomento de la captación de energía mediante otro tipo de fuentes, las renovables. Dentro de las energías renovables la solar es quizás las más destacada según el European Solar Thermal Industry Federation (1). Por ello se hace relevante el análisis y la valoración de los sistemas actuales de captación solar térmica (de aquí en adelante CST) para descubrir sus limitaciones y estudiar mejoras e innovaciones que permitan promover un mayor uso ${ }^{1}$. ¿Cuáles son estos aspectos a mejorar?

\section{REQUERIMIENTOS PARA EL DISEÑO DE UN NUEVO CST}

Son bien conocidos los problemas de integración en la arquitectura de estos elementos captadores, casi siempre relegados a zonas de cubierta donde no se note su presencia. Por tanto, se precisa de un sistema CST capaz de integrarse arquitectónicamente ${ }^{2}$ en las envolventes edificatorias. Para ello es importante que el nuevo sistema captador ofrezca capacidad de juego combinatorio con el resto del cerramiento y presente variedad de acabados. La presencia de los materiales cerámicos pueden en este punto tomar un papel importante.

Es necesario que el nuevo sistema captador cumpla una doble funcionalidad: energética y constructiva. En términos constructivos, el sistema captador tiene que formar parte de la propia envolvente del edificio (fachada y/o cubierta) y garantizar, como mínimo, las mismas propiedades que el cerramiento tradicional al que sustituye. Debe ser capaz de ser instalado en fachada y/o cubierta sin la necesidad de recurrir a una subestructura auxiliar, aprovechando la propia subestructura de la envolvente del edificio, de manera que simplifique el replanteo en obra, que ofrezca la posibilidad de regular fácilmente su planeidad, que garantice una ejecución segura, rápida y sencilla, que favorezca los rendimientos de ejecución y que permita una fácil sustitución o extracción de las piezas en caso de rotura o avería.

Con esta integración del sistema captador en la envolvente del edificio, cualquier parte de la fachada puede ser captadora, su integración arquitectónica permitiría ser colocado en grandes superficies y, de esta manera, se posibilitaría la oportunidad de trabajar a temperaturas más bajas que las de los sistemas convencionales, evitando los problemas de sobrecalentameinto de los captadores convencionales existentes actualmente que inducen a establecer sistemas de disipación energética, mantenimiento y regulación de temperatura. Porque si nos atenemos al clima mediterráneo u otro clima similar es suficiente y adecuado diseñar un sistema que obtenga unas temperaturas de trabajo comprendidas entre los 30 y $60^{\circ} \mathrm{C}$.
También se requiere un sistema que sea de mayor viabilidad económica para el usuario final y para ello deberían evitarse el uso de chapas y conductos metálicos o los recubrimientos especiales que encarecen los sistemas de captación. Es necesario, entonces, diseñar un CST formado por materiales más económicos, como los cerámicos, que hoy en día podría sustituir a los metales en estas aplicaciones. En la misma dirección, para evitar la colocación de la cara caja de chapa de acero hermética ${ }^{3}$ en cada captador resultaría más apropiado usar materiales aislantes de panel rígido, poro cerrado (impermeables al agua) y capaces de soportar altas temperaturas de servicio.

Por último, el proceso de conexionado debe ser fácil, seguro y que no requiera de mano de obra especializada, es decir, evitando la presencia del fontanero en fachada.

Todos estos aspectos se introducen como objetivos para proponer un nuevo sistema captador solar térmico: realizado principalmente con materiales cerámicos, integrado arquitectónicamente formando parte de la misma envolvente del edificio y adecuado su rendimiento al clima mediterráneo o similar.

\section{PROPUESTA DE UN NUEVO SISTEMA DE CAPTACIÓN SOLAR TÉRMICA}

\subsection{Configuración general: formatos y materiales}

A partir de los requerimientos anteriores se establece un sistema de envolvente cerámica formado por dos elementos principales: un panel captador cerámico con cubierta de vidrio y un panel no captador cerámico sin vidrio (Figura 1). De esta manera se obtiene una adecuada combinación de superficies brillantes (paneles captadores) con superficies no brillantes (paneles no captadores) que ayuda, además, a concertar compositivamente con las superficies brillantes o reflectantes dadas en un edificio como las ventanas, vidrieras, lucernarios, etc.

Para estos paneles se proponen dos tipos de formatos distintos para aumentar la versatilidad del sistema: uno de dimensión menor de $1.000 \times 1.000 \mathrm{~mm}$ y otro mayor de 1.000 $\times 3.000 \mathrm{~mm}$. El panel de mayor formato consigue una gran velocidad de montaje del sistema y una ejecución con menores conexiones y juntas en la envolvente. Sin embargo, sus recambios serán más caros y se requerirá de medios auxiliares para su correcto manejo y colocación. Mientras que el formato pequeño muestra una más fácil manipulación en obra y permite adaptarse con mayor facilidad a los huecos de ventana, pero presenta mayores conexiones por superficie de envolvente.

Una vez establecida la configuración general del nuevo CST, se realiza un primer bloque de ensayos basados principalmente en la elección material y sus espesores correspondien-

\footnotetext{
Este artículo parte del proyecto de investigación: «Colectores solares térmicos cerámicos. Integración arquitectónica», presentado por la empresa Uralita Iberia, el Instituto de Tecnología Cerámica (ITC) y la Universitat Internacional de Catalunya (UIC Barcelona). Este proyecto obtuvo una subvención del Ministerio de Industria, Turismo y Comercio en la convocatoria de ayudas del Plan Nacional de Investigación Científica, Desarrollo e Innovación Tecnológica 2008-2011, con n. ${ }^{0}$ de expediente: IAP-590000-2008-11.

${ }^{2}$ Se entiende por integración arquitectónica a aquel elemento que es capaz de inhibirse estéticamente o destacar en igual intensidad que el resto de elementos de una fachada o cubierta.

${ }^{3}$ Las cajas metálicas herméticas de los colectores convencioanles, además de ser utilizadas como elemento estanco y de protección del aislante térmico de poro abierto y otros materiales, se utiliza, en algunos casos, como elemento de agarre y sujeción a la subestructura auxiliar.
} 


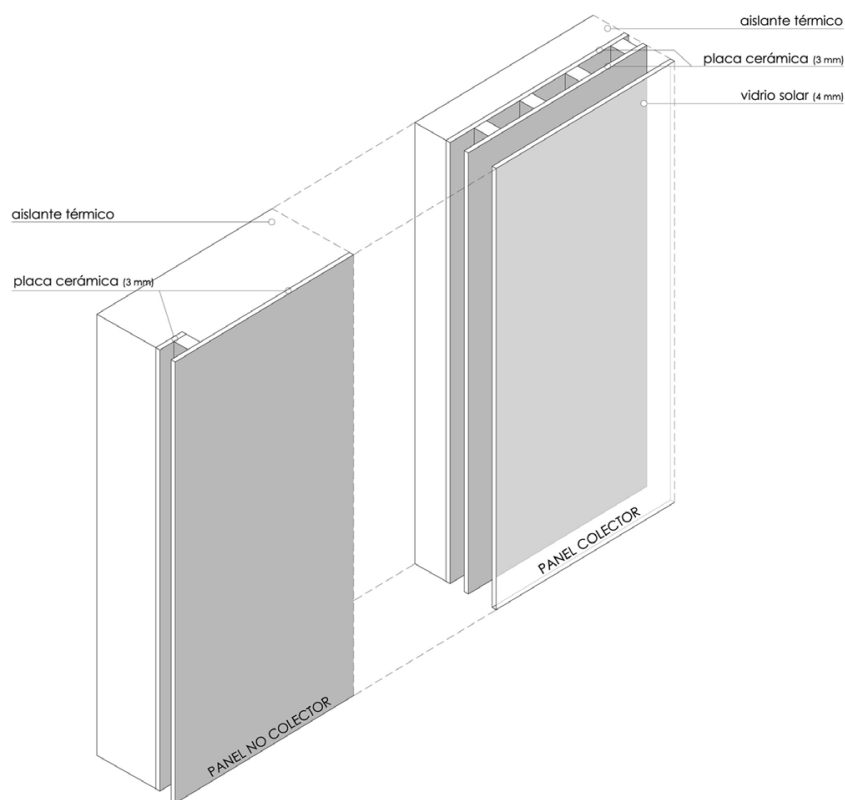

Figura 1. Configuración del modelo general de panel colector y panel no colector (Roviras, J., 2012).

tes. Se pretende, entonces, definir los espesores de las cámaras interiores (la trampa de infrarrojos y el circuito interno para el paso del líquido caloportador) y de los propios materiales que conforman el panel colector (placa absorbente cerámica, aislante térmico y vidrio). Además, al mismo tiempo, se analiza mediante la construcción de pequeños prototipos el rendimiento energético del captador y el sistema de fijación e instalación de los paneles en fachada.

\subsubsection{La cerámica}

Como ya se ha comentado anteriormente, se ha buscado que la cerámica juegue un papel fundamental en el diseño de este sistema. Pero para ello es preciso utilizar un producto que sea capaz de adaptarse a las necesidades técnicas, arquitectónicas y energéticas del proyecto y se ha encontrado en una losa porcelánica de gran superficie y pequeño espesor, llamada comercialmente Laminam $^{4}$. Se trata de un formato con unas dimensiones de ancho y largo de $1.000 \times 3.000 \mathrm{~mm}$ y $3 \mathrm{~mm}$ de espesor que brinda una gran variedad de acabados y colores tanto para ofrecer una gama arquitectónica interesante como para conseguir absortancias rendibles en los colores más oscuros (2). Debido a su amplia superficie posibilita configuraciones de colector en paneles de forjado a forjado y debido a su reducido espesor presenta un peso muy reducido (Figura 2) y permite ser cortado ${ }^{5}$ con gran facilidad y precisión para configurar los tabiquillos interiores del colector.

\subsubsection{El vidrio}

Para mejorar el rendimiento del colector cerámico es necesario trabajar con un vidrio especial (vidrio solar) de elevada transmisividad, debido a su bajo contenido en $\mathrm{FE}_{2} \mathrm{O}_{3}$, muy utilizado en los captadores comercializados. Al prever la colocación de los colectores en fachada es preciso trabajar con vidrios laminados y para evitar excesivos pesos se ha escogido un espesor de $4 \mathrm{~mm}$ (3).

\subsubsection{El adhesivo}

El reto principal en este apartado ha sido encontrar un tipo de adhesivo que permita unir la placa cerámica base con el absorbedor también cerámico y este último con el vidrio. Por tanto, tenemos dos tipos de uniones que resolver: cerámicacerámica y cerámica-vidrio.

Por un lado, el adhesivo empleado en la unión entre el vidrio y la cerámica deberá ser resistente a los agentes atmosféricos y a la radiación ultravioleta, de modo que sus prestaciones no se deterioren con esa exposición. Deberá resistir también los esfuerzos de cizalla derivados del peso de la lámina de vidrio. A fin de conocer estos esfuerzos a que estará sometido el material adhesivo en esta unión se ha realizado un modelo de simulación. Para ello se ha considerado que la capa de adhesivo tiene un espesor de $2 \mathrm{~mm}$ y una anchura de $20 \mathrm{~mm}$.

Por otro lado, el adhesivo a emplear como elemento de unión entre el absorbedor y la placa cerámica base (unión cerámicacerámica) debe ser resistente al agua y al cloro. Además, debe
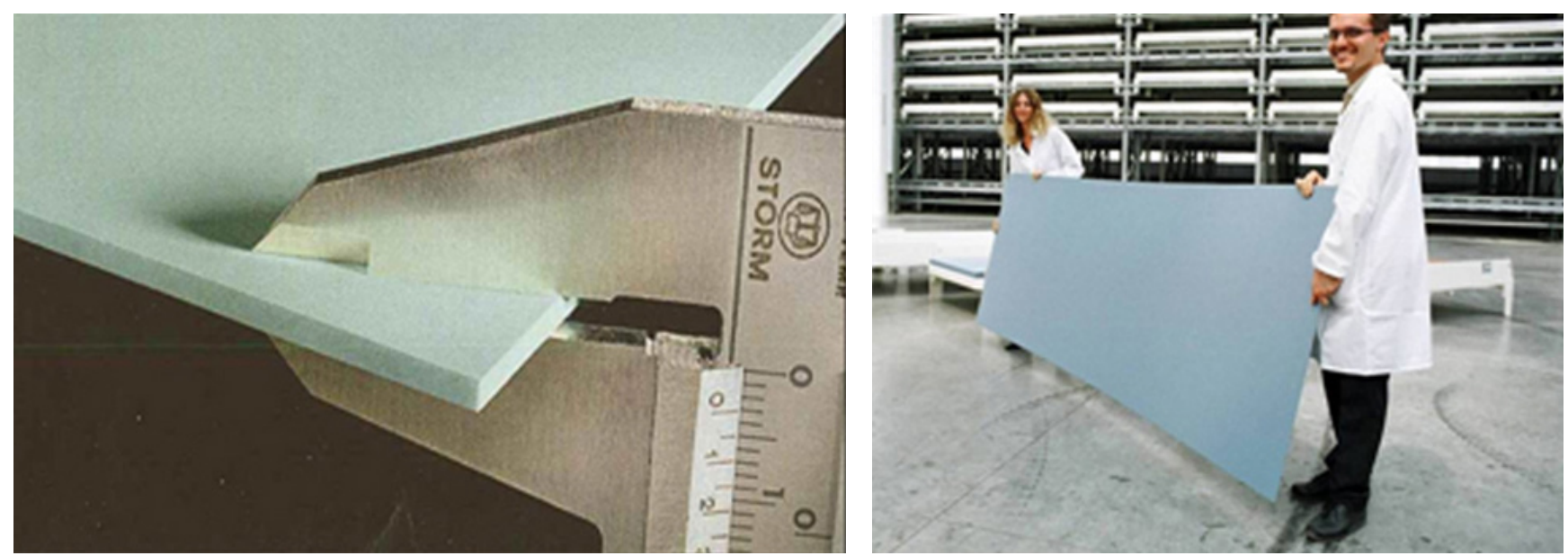

Figura 2. Losa cerámica porcelánica (Laminam, 2010).

\footnotetext{
4 El producto Laminam nace en 2001 de la idea del ingeniero Franco Stefani.

${ }_{5}^{5}$ El corte se realiza a mano y el utensilio es un simple corta-vidrios de metal con rulina autonivelante.
} 
presentar una resistencia a la tracción elevada ya que en la cavidad definida entre ambas placas cerámicas y los tabiques separadores circula agua a una determinada presión. También se debe teber en cuenta el rango de temperaturas en que el adhesivo mantiene sus propiedades, que debe estar comprendido dentro del rango de trabajo del captador, ya que en ocasiones se pueden alcanzar temperaturas relativamente elevadas (hasta $100^{\circ} \mathrm{C}$ ). Los adhesivos suministrados por las empresas especializadas en este tipo de unión (cerámica-cerámica) han sido sometidos al ensayo de tracción en laboratorio. Para ello se han cortado piezas cerámicas rectangulares de $20 \times 65 \mathrm{~mm}$ y se han pegado en cruz con cada uno de los adhesivos estudiados con un área de unión de $20 \times 20 \mathrm{~mm}^{2}$. Las piezas se han mantenido pegadas durante tres días, para asegurar un completo curado del material adhesivo. Previa realización del ensayo, las piezas pegadas se han mantenido sumergidas en un baño termostático de agua en ebullición, es decir, a $100{ }^{\circ} \mathrm{C}$, a fin de que la unión se encontrara en unas condiciones de temperatura y humedad exigentes.

\subsubsection{El aislante térmico}

En este apartado se han analizado la gran mayoría de aislantes térmicos existentes en el sector de la edificación. De cada uno de ellos se analiza su resistencia al agua, su capacidad de aportar rigidez al panel colector, su temperatura máxima de servicio, su conductividad térmica y finalmente su coste (Tabla 1).

Después de este análisis el material escogido por reunir las condiciones básicas antes planteadas es el panel rígido de poliisocianurato (PIR). Se trata de un panel fabricado en línea continua, dispuesto en sándwich, formado por un núcleo o centro de espuma rígida de poliisocianurato y dos caras de papel de diversos acabados. Los formatos que presenta son amplios, partiendo de los $1.220 \mathrm{~mm}$ de anchura y pudiendo llegar hasta los $6.000 \mathrm{~mm}$ de longitud. La conductividad térmica que presenta este material es muy baja (alrededor de los $0,021 \mathrm{~W} / \mathrm{mK}$ ) y es capaz de soportar una temperatura de servicio máxima de $121{ }^{\circ} \mathrm{C}$. Además, su precio de mercado se encuentra en unos rangos muy parecidos a los de la lana de roca.

\subsection{Campaña de ensayos}

Una vez ya escogidos y analizados los distintos materiales que componen el nuevo colector, se ha procedido a la realización de una campaña de ensayos que han permitido conocer, entre otros aspectos, la distancia óptima entre la lamina de vidrio y el absorbedor cerámico (espesor de la trampa de infrarrojos), la resistencia a la presión, dilatación y estanqueidad del captador, la compatibilidad entre materiales, la caracterización del circuito interior (serie/ paralelo), la definición del espesor, anchura y separación de los tabiques cerámicos interiores que definen el circuito interior, el tipo de conducto y el sistema de conexionado. De cada uno de estos ensayos, presentados en la tesis doctoral de Roviras (4), pero que por su extensión no han podido ser publicados en este artículo, se han determinado los siguientes parámetros:

- El espesor óptimo de la trampa de infrarrojos se define en $10 \mathrm{~mm}$.

- Las pruebas de presión del captador definen una anchura de cavidad (distancia entre tabiques interiores por la que circulará el líquido caloportador) de $50 \mathrm{~mm}$ como máximo. Y los ensayos de dilatación y estanqueidad verifican que los prototipos ensayados, a distintas temperaturas y presiones, no han presentado fugas. Tampoco se han observado formación de fisuras por la dilatación térmica.

- El estudio de compatibilidad entre materiales realizado mediante el análisis de las dilataciones diferenciales existentes entre los distintos materiales que forman el colector, verifica que el captador resiste sin problemas los cambios bruscos de temperatura a los que pueda estar sometido.

- Según la caracterización del circuito interno (por el que circulará el líquido caloportador), el colector con circuito interno en paralelo presenta un rendimiento ligeramente superior al del colector con circuito en serie.

- De todos los conductos estudiados, los modelos de polietileno reticulado (PEX) se consideran los más adecuados. Se establece como óptimo un diámetro interior de conducto de $12-13 \mathrm{~mm}$.

Tabla 1. Valores analizados de cada uno de los aislantes térmicos (Roviras, J., 2013).

\begin{tabular}{|l|c|c|c|c|}
\hline \multicolumn{1}{|c|}{ Material aislante } & $\begin{array}{c}\text { Conductividad } \\
\text { térmica }(\mathbf{W} / \mathbf{( m K )})\end{array}$ & $\begin{array}{c}\text { Empresa } \\
\text { suministradora }\end{array}$ & Coste (€/m²) & Observaciones \\
\hline Lana de roca & 0,037 & Rockwool & $8-13 € / \mathrm{m}^{2}$ & Sensible al agua y/o humedad \\
\hline Aerogel & 0,014 & Aspen & $42 € / \mathrm{m}^{2}$ & Precio demasiado alto \\
\hline Corcho proyectado & 0,037 & Paymo & - & $\begin{array}{c}\text { Espesor proyectable } 20 \text { mm. No es } \\
\text { rígido }\end{array}$ \\
\hline Poliestireno extrudido (XPS) & 0,035 & Dow & $7-15 € / \mathrm{m}^{2}$ & $\begin{array}{c}\text { Temperatura máxima de servicio: } \\
75^{\circ} \mathrm{C}\end{array}$ \\
\hline $\begin{array}{l}\text { Poliestireno extrudido con } \\
\text { grafito }\end{array}$ & - & Cupa group* & - & Uso exclusivo para Thermoslate \\
\hline $\begin{array}{l}\text { Proyectado de } \\
\text { poliisocianurato }\end{array}$ & 0,021 & Soltech Energy* & - & $\begin{array}{c}\text { Temperatura máxima de servicio: } \\
110^{\circ} \mathrm{C}\end{array}$ \\
\hline $\begin{array}{l}\text { Paneles rígidos de } \\
\text { poliisocianurato (PIR) }\end{array}$ & 0,026 & Europerfil & $5-9 € / \mathrm{m}^{2}$ & $\begin{array}{c}\text { Temperatura máxima de servicio: } \\
120^{\circ} \mathrm{C}\end{array}$ \\
\hline $\begin{array}{l}\text { Poliestireno expandido con } \\
\text { grafito (EPS) }\end{array}$ & 0,032 & Basf & - & $\begin{array}{c}\text { Baja temperatura de servicio. } \\
\text { Máxima: } 35^{\circ} \mathrm{C}\end{array}$ \\
\hline Resina de melamina & - & Basf & - & - \\
\hline $\begin{array}{l}\text { Tableros rígidos de fibra de } \\
\text { madera }\end{array}$ & 0,037 & Gutex & $18-22 € / \mathrm{m}^{2}$ & $\begin{array}{c}\text { Resistente a altas temperaturas. } \\
\text { Precio demasiado alto }\end{array}$ \\
\hline
\end{tabular}

* Fabricantes de colectores solares térmicos que también producen su propio aislante térmico. 



Figura 3. Dispositivo experimental utilizado y panel colector cerámico construido para el ensayo (ITC 2012).

\subsection{Caracterización del colector solar cerámico}

Para validar la configuración del panel colector cerámico propuesto se ha comprobado la viabilidad energética del mismo según la normativa aplicable a captadores de energía solar térmica (5). En este ensayo ha sido necesario construir un prototipo de $1.000 \times 1.000 \mathrm{~mm}$ utilizando los materiales y resultados obtenidos en los ensayos anteriores, orientado a Sur e instalado con una inclinación de $40^{\circ}$ sobre la horizontal. Para la obtención de datos energéticos se ha utilizado un dispositivo experimental monitorizado y específico basado principalmente en un baño termostatado, una bomba de membrana, un caudalímetro, tres termorresistencias, un piranómetro y un anemómetro (Figura 3).

Es importante destacar que la curva característica de un CST (Tabla 4) se extrae de tres parámetros que proporcionan información sobre el comportamiento térmico del mismo. En primer lugar se encuentra la eficiencia óptica $\left(\left.\mu\right|_{\mathrm{T}^{*}=0}\right)$ : que es el valor de la ordenada en el origen de la recta y corresponde al valor del rendimiento del captador cuando el parámetro $\mathrm{T}^{*}$ es igual a cero $^{6}$, es decir la temperatura de entrada del agua $\left(\mathrm{T}_{\mathrm{e}}\right)$ es igual a la temperatura ambiente $\left(\mathrm{T}_{\mathrm{a}}\right)$. Éste es el máximo rendimiento que se puede obtener con un captador. Cuanto más alto sea, mejor será el comportamiento térmico del mismo. Por otro lado tenemos el Coeficiente gobal de pérdidas: siendo éste el valor de la pendiente de la curva característica y hace referencia a las pérdidas de calor del captador, cuanto mejor aislado y diseñado está un captador menor será el valor de este parámetro. $\mathrm{Y}$, por último, la temperatura de estancamiento $\left(\left.\mathrm{T}^{*}\right|_{\mu=0}\right)$ : es el valor del parámetro $\mathrm{T}^{*}$ para el que el rendimiento del captador es nulo, es decir, el captador no puede elevar la temperatura del agua que está entrando para unas condiciones ambientales dadas. Cuanto mayor sea la eficiencia óptica y/o menor sea el coeficiente global de pérdidas, mayor será el valor de este parámetro. Por tanto, la eficiencia energética de un colector será mayor cuanto mayor sea su eficiencia óptica y menor sea su coeficiente global de pérdidas.

Los parámetros medidos durante el ensayo han presentado respuestas positivas. En las gráficas de los resultados obtenidos se observa una adecuada relación entre la radiación solar incidente y la temperatura de salida del agua del colector (Tabla 3), que llega a una punta máxima de $32{ }^{\circ} \mathrm{C}$, suficiente para el rendimiento buscado ${ }^{7}$. Con estos datos recogidos durante el ensayo (Tabla 2), se ha definido la curva característica del colector cerámico ensayado y se ha comparado junto con las curvas de otros dos captadores comerciales, uno de óptimas prestaciones y otro de características mínimas: la curva del colector propuesto se encuentra dentro del rango de rendimiento habitual para captadores comerciales (Tabla 4). En cualquier caso hay que tener en cuenta que el colector óptimo considerado (CST convencional) emplea materiales metálicos en su construcción (mejor conductor térmico que la cerámica), recubrimientos especiales y selectivos que poseen

Tabla 2. Valores de la eficiencia óptica, coeficiente global de pérdidas y temperatura de estancamiento del captador cerámico y de dos captadores comerciales (ITC 2012).

\begin{tabular}{|l|c|c|c|}
\hline & $\begin{array}{c}\text { Eficiencia óptica } \\
\boldsymbol{\mu} \mid \mathbf{T}^{*}=\mathbf{o}(\mathbf{\%})\end{array}$ & $\begin{array}{c}\text { Coeficiente global } \\
\text { de pérdidas } \\
\left(\mathbf{W} / \mathbf{m}^{2} \mathbf{C}\right)\end{array}$ & $\begin{array}{c}\text { Temperatura de } \\
\text { estancamiento } \\
\mathbf{T}^{*} \mid \boldsymbol{\mu}=\mathbf{0}\left({ }^{\circ} \mathbf{C m}^{2} / \mathbf{W}\right)\end{array}$ \\
\hline Captador cerámico & 75,9 & 7,98 & 0,097 \\
\hline Captador comercial mínimo & 65,0 & 8,00 & 0,081 \\
\hline Captador óptimo & 82,0 & 4,00 & 0,205 \\
\hline
\end{tabular}

\footnotetext{
${ }^{6}$ Siendo $\mathrm{T}^{*}$ la función de la temperatura de entrada del agua $\left(\mathrm{T}_{\mathrm{e}}\right)$, la temperatura ambiente $\left(\mathrm{T}_{\mathrm{a}}\right)$ y la radiación solar $\left(\mathrm{G}_{\mathrm{s}}\right)$, mediante la siguiente ecuación: $T^{*}=T_{e}-T_{a} /$ Gs. Por tanto, la determinación de la curva característica consiste en conocer la relación $\mu=\mu\left(T^{*}\right)$.

Recordemos que para un CST como el propuesto, en un clima mediterráneo y con una integración arquitectónica que permitiera ser colocado en grandes superficies, se posibilitaría la oportunidad de trabajar a temperaturas más bajas que las de los sistemas convencionales y, por tanto, resultarían suficientes temperaturas comprendidas entre los 30 y $60{ }^{\circ} \mathrm{C}$.
} 
Tabla 3. Valores de calentamiento del líquido caloportador durante un día (ITC 2012).

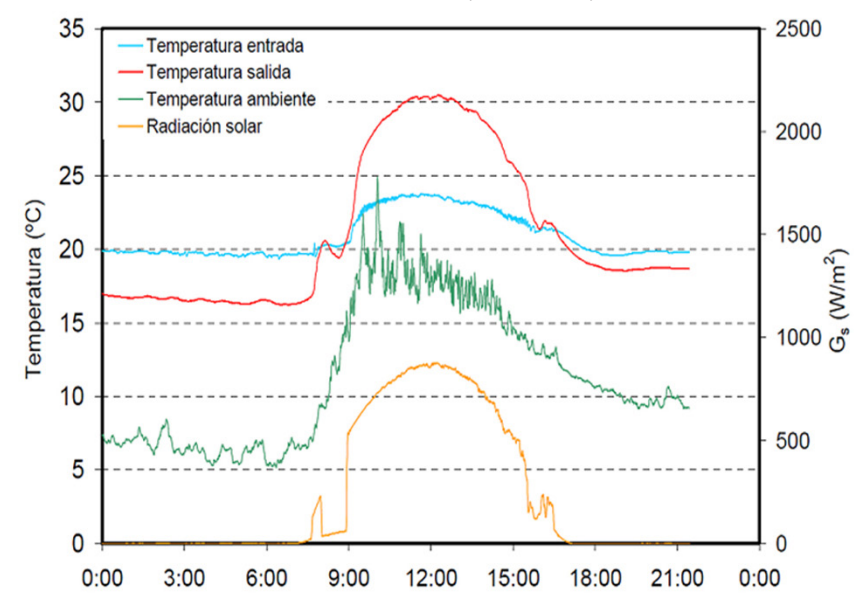

una alta absortividad de la radiación solar y que reducen las pérdidas del captador. Los datos obtenidos con el captador cerámico son razonablemente buenos teniendo en cuenta los materiales utilizados y la gran superficie de la que puede disponer (toda la fachada) al tratarse de un sistema integrado que forma parte del sistema de cerramiento exterior de un edificio (6).

\subsection{Propuesta de diseño del sistema constructivo}

Paralelamente a los ensayos anteriores, se ha trabajado en el diseño del sistema constructivo para la instalación de la envolvente cerámica captadora que, para conseguir una plena integración arquitectónica, debe formar parte de la propia envolvente del edificio y responder a las mejoras propuestas en la introducción de este escrito, tal como se muestra también en los trabajos de Luis Juanicó (7): facilidad de replanteo, rapidez de ejecución, seguridad en el montaje para el operario, facilidad de desmontaje de los paneles de forma individual y prioridad del montaje desde el interior.

Además de los aspectos anteriores, cabe añadir que el sistema constructivo debe ser capaz de absorber posibles movimientos estructurales, actuar independientemente de la hoja interior para evitar la existencia de puentes acústicos y ofrecer un espesor final del cerramiento (envolvente captadora + soporte + acabado interior) no superior a los $30 \mathrm{~cm}$ para poder ser competitivo en el mercado actual. Bajo estas premisas se desarrolla un sistema constructivo con un total de cinco perfiles metálicos distintos, tres de ellos en formatos existentes en el mercado actual (encontrados en los prontuarios básicos de perfiles metálicos). Este último aspecto repercute muy favorablemente en el coste final del sistema.

Se describen a continuación los 8 pasos a realizar para la ejecución del cerramiento (Figura 4):

Paso 1: Aplomado de un perfil angular perimetral

Este primer paso parte de la estructura de pilares y forjados ya construidos, colocando un angular perimetral de forjado (perfil en «L») alineado con la plomada definida en obra. Dicho perfil se ancla al forjado mediante tornillería a través de unos agujeros colisos provistos ya en el propio perfil para adaptar su alineación a la plomada. Todo ello realizado desde el interior del edificio. Seguidamente, se coloca una
Tabla 4. Curva característica que define el rendimiento del colector solar cerámico (ITC 2012).

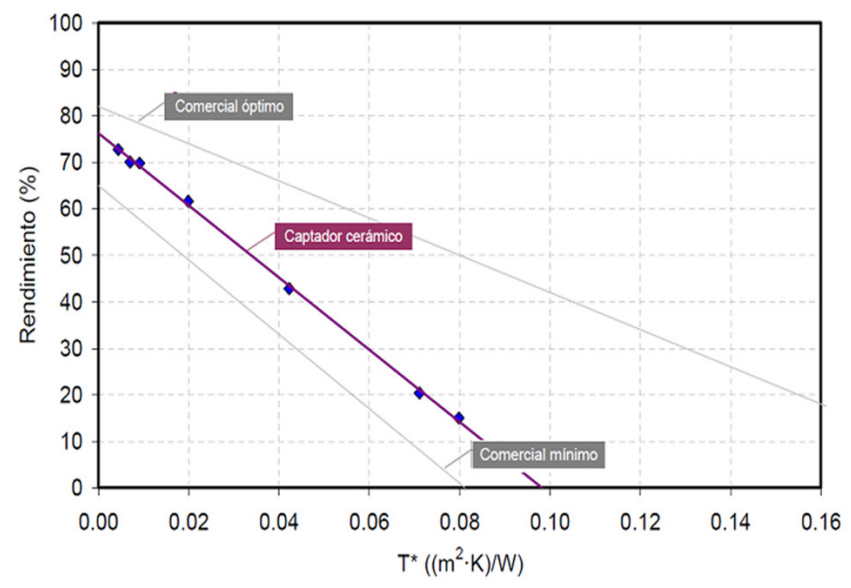

lámina desolidarizadora pasándola por encima del angular perimetral y preparada para recibir la base regularizadora de mortero autonivelante para el pavimento de acabado. El pliegue vertical del angular perimetral actúa también en este caso como elemento de contención de esta capa regularizadora.

Paso 2: Fijación de montantes verticales

El siguiente paso fija los montantes verticales de chapa de acero galvanizado de perfil $\Omega 100$ al angular perimetral en $\mathrm{L}$. La sujeción de los montantes se realiza a través de las alas del perfil omega mediante tornillería específica. La altura total de este perfil es variable, en función de la altura existente entre forjados del edificio. La parte superior de los montantes verticales queda alineada a una altura específica y controlada mediante láser para garantizar el posicionamiento homogéneo de todos los perfiles. Se define una altura de unos $70 \mathrm{~cm}$ por encima del nivel de forjado ya regularizado que asegura una posición cómoda y segura de trabajo del operario en la conexión entre perfiles. El resto de longitud del perfil, hasta llegar a los $3 \mathrm{~m}$, o aproximado, quedará siempre por debajo esperando ser conectado al perfil inferior mediante pieza conectora. Esta operación también se realiza desde el interior del edificio.

\section{Paso 3: Conexión entre montantes verticales}

Posteriormente se procede al conectado de los perfiles verticales $(\Omega 100)$ mediante un elemento conector de acero galvanizado también de sección omega. Dicho conector se coloca con seguridad y comodidad desde el interior del edificio y garantiza la unión entre montantes verticales, además de actuar como elemento regularizador de las alturas entre forjados. La unión se realiza mediante tornillería específica a través de agujeros colisos ya existentes en el perfil conector. De esta manera, mediante los colisos, se resuelve el problema generado por la dilatación térmica de los perfiles, obteniendo la holgura necesaria entre perfil y perfil que evita los esfuerzos que se podrían producir por la dilatación de los mismos.

\section{Paso 4: Fijación de correas horizontales}

Una vez anclados y conectados todos los montantes verticales se procede a la colocación de las correas horizontales como 


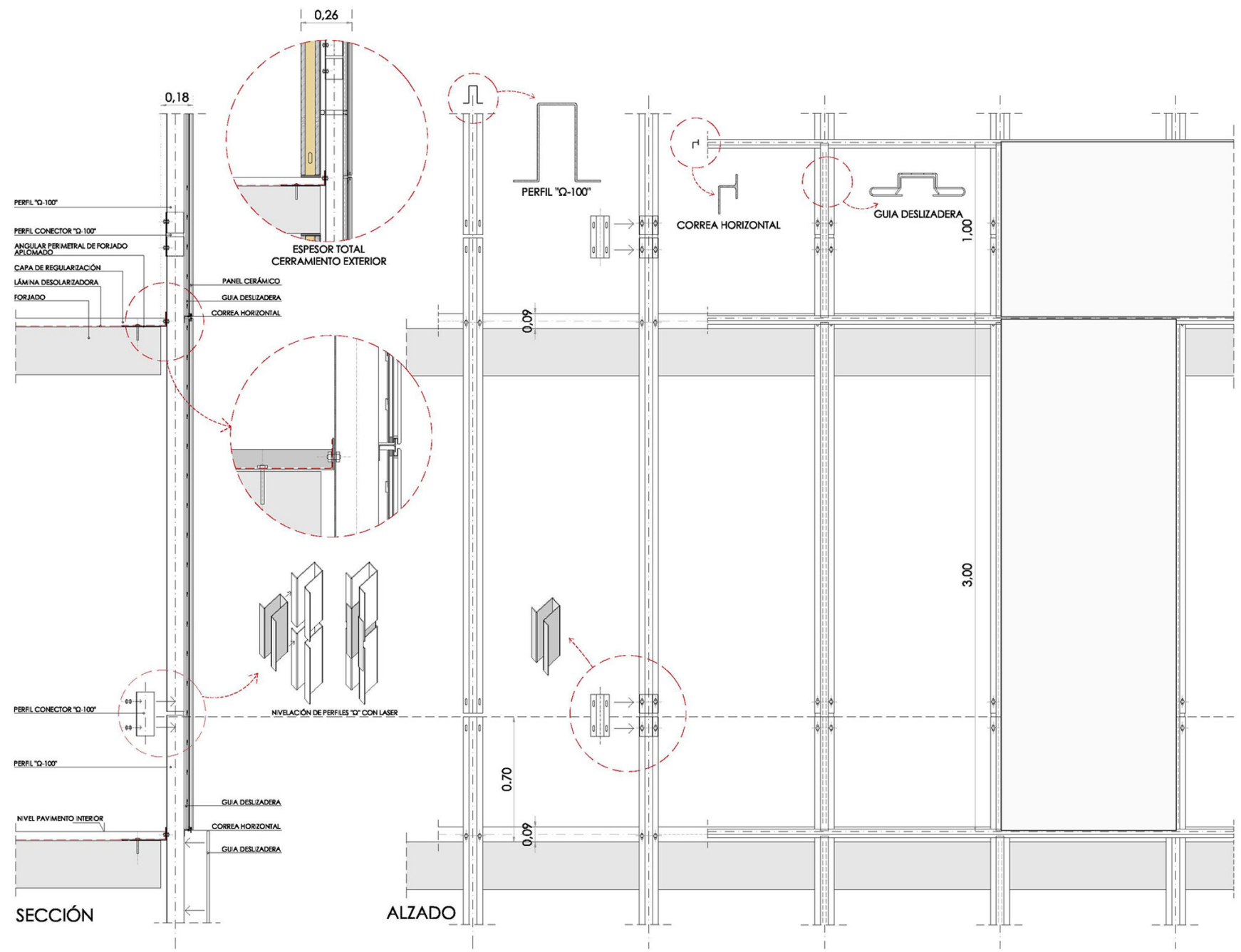

Figura 4. Secuencia de montaje de la estructura metálica. Detalle derecha: sección del cerramiento que define un espesor total de 26 cm (Roviras, J., 2013).

guías de soporte para los paneles colectores y no colectores. Su replanteo y situación sigue la modulación establecida según el formato de los paneles.

Paso 5: Fijación de las guías deslizaderas ${ }^{8}$

Las guías deslizaderas, encargadas de sujetar los paneles en todo el largo de sus dos lados verticales, se fijan directamente a la parte más exterior del montante vertical mediante tornillería. Dichas guías irán colocadas siempre entre correas horizontales.

Pasos 6 y 7: Fijación y conexionado de los paneles

La colocación de los paneles sigue la siguiente secuencia (Figura 5): primero se encaja el panel en la correa horizontal superior y oscila hacia el interior hasta encontrar la guía inferior. Seguidamente se desliza sobre esta correa horizontal inferior hasta unirlo con su vecino, de manera que el panel se sostenga por sí solo entre las correas horizontales. Poste- riormente se fijan a los anclajes preparados en los montantes verticales. Por último, se instalan y conectan las tuberías de los paneles colectores, todo desde el interior.

Esta secuencia de montaje ofrece la posibilidad de un fácil y rápido desmontaje de los paneles en caso de avería, reparación o rotura de alguno de ellos, sin la necesidad de intervenir o manejar en los paneles vecinos. En este sentido ha sido necesario estudiar la sección del panel y prever un espesor mínimo de las juntas horizontales que permitan el juego de movimientos necesarios del panel como para que éste pueda ser descolgado con facilidad.

\section{Paso 8: Cerramiento de tabiquería interior}

Una vez realizado todo el conexionado de los paneles colectores, se procede a la ejecución del trasdosado interior con cualquiera de los sistemas existentes en el mercado de tabiquería industrializada de placas de yeso laminado y perfiles de chapa de acero.

\footnotetext{
${ }^{8}$ Este sistema de fijación surge del proyecto de investigación GLACTIS, con número de referencia DEX-5300oo-2008-91 obtuvo una financiación pública del Estado español a través del Ministerio de Industria, Turismo y Comercio y se desarrolló también juntamente con la Universitat Internacional de Catalunya (UIC), Uralita Iberia y la empresa de materiales cerámicos Saloni.
} 


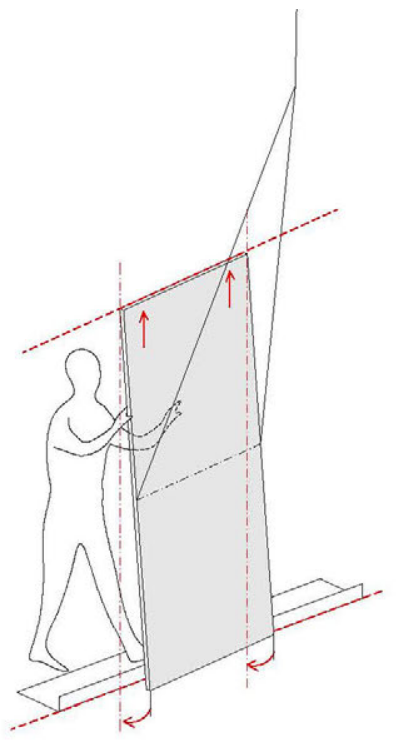

ENCAJE SUPERIOR OSCILACION INFERIOR

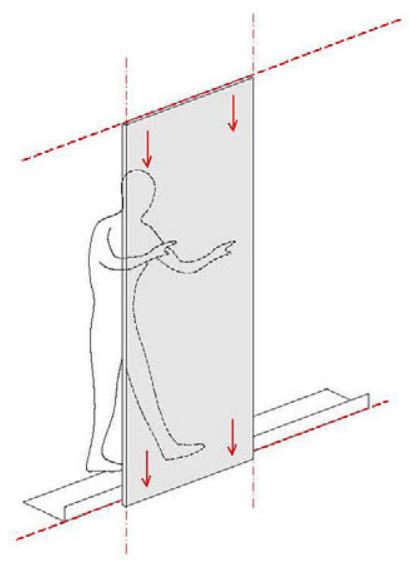

APOYO INFERIOR
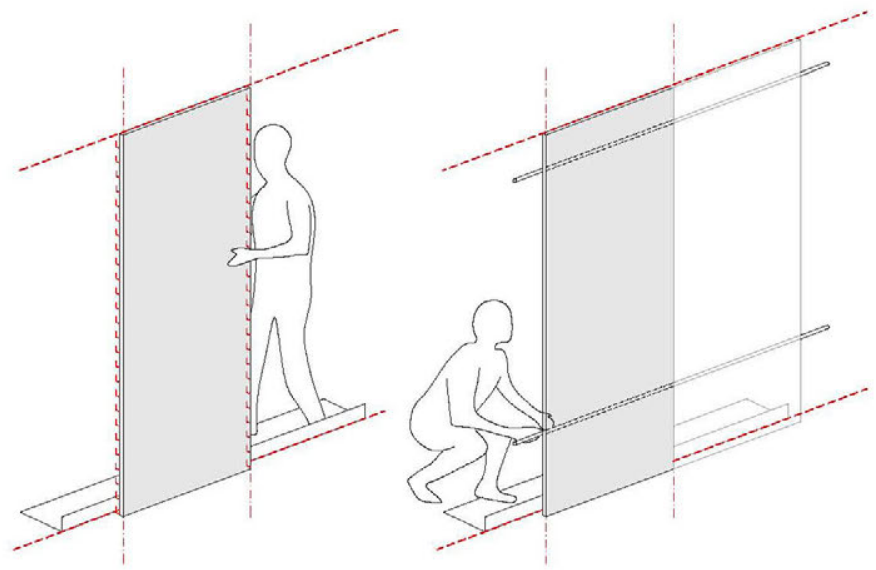

FIJACIÓN FRONTAL
PASO Y CONEXION

DE TUBOS

Figura 5. Secuencia de colocación de los paneles cerámicos colectores y no colectores sobre la estructura diseñada (Roviras, J., 2013).

Con este diseño se consigue que el sistema ofrezca una doble funcionalidad tanto energética como constructiva, garantizando los mismos requerimientos y espesores (no mayores de $26 \mathrm{~cm}$ ) que un cerramiento convencional pero con un captador solar integrado dentro de este espesor.

\section{CONSTRUCCIÓN DE PROTOTIPOS Y ENSAYO REAL}

Finalmente se han construido un conjunto de prototipos, cuatro paneles captadores y dos paneles no captadores (Figura 6), con el objetivo de construir una porción de fachada para evaluar su rendimiento energético, su viabilidad técnica-constructiva y su integración arquitectónica ${ }^{9}$.

Previamente al montaje se tuvieron que realizar dibujos específicos sobre la ejecución de la fachada prototipo que aseguraran la máxima precisión de la ejecución tanto de la estructura metálica como de los propios paneles cerámicos (Figura 7).

Con los colectores ya colocados sobre la subestructura metálica se observa con detalle el correcto encaje de las distintas piezas y el buen comportamiento del sistema en general. La planeidad superficial de los colectores es prácticamente perfecta y no se detectan desajustes importantes entre los mismos ${ }^{10}$.

Para determinar la eficiencia de los colectores solares cerámicos se ha utilizado un montaje experimental específico. La evolución de algunos de los parámetros obtenidos (temperatura ambiente, radiación solar y temperatura de entrada y salida del agua) a lo largo de un día típico y soleado de febrero pre- sentan un notable interés y validan el buen comportamiento del captador en momentos de incidencia solar sobre los mismos. Es importante destacar que al colocar los paneles captadores en vertical se reduce su rendimiento energético, pero se compensa dicho aspecto con la incorporación de más paneles captadores, al disponer de toda la superficie de fachada (8).

Una vez finalizados los ensayos energéticos y transcurridos 18 meses desde la instalación de esta fachada prototipo, se ha verificado el buen comportamiento del sistema de anclaje, no se han detectado defectos o patologías apreciables y tanto los paneles captadores como los no captadores presentan un muy buen estado (Figura 8).

\section{HERRAMIENTA MULTICRITERIO PARA LA EVALUACIÓN DEL CST CERÁMICO}

\subsection{Introducción}

Una vez demostrada la viabilidad técnico-arquitectónica y energética del nuevo CST cerámico, se define una herramienta que permite evaluar desde un punto de vista multicriterio (coste, impacto social e impacto ambiental) el nuevo colector, en comparación con los colectores comerciales existentes en la actualidad.

\subsection{Método}

La herramienta definida se basa en la teoría de utilidad multiatributo (9) considerada como la técnica de ayuda a la decisión multicriterio más adecuada entre las cinco principales

\footnotetext{
9 Dicho ensayo de campo se realiza en la cubierta del laboratorio del ITC en Castellón, zona con condiciones típicas de clima mediterráneo, con altos índices de radiación solar durante todo el año.

${ }^{10} \mathrm{El}$ sistema constructivo aquí presentado es válido para la ejecución de dicha envolvente cerámica captadora tanto en nueva edificación como en edificación existente con pequeñas variaciones únicamente en el sistema de conexionado de los paneles (paso 7). Este caso podría ser objeto de otro artículo.
} 


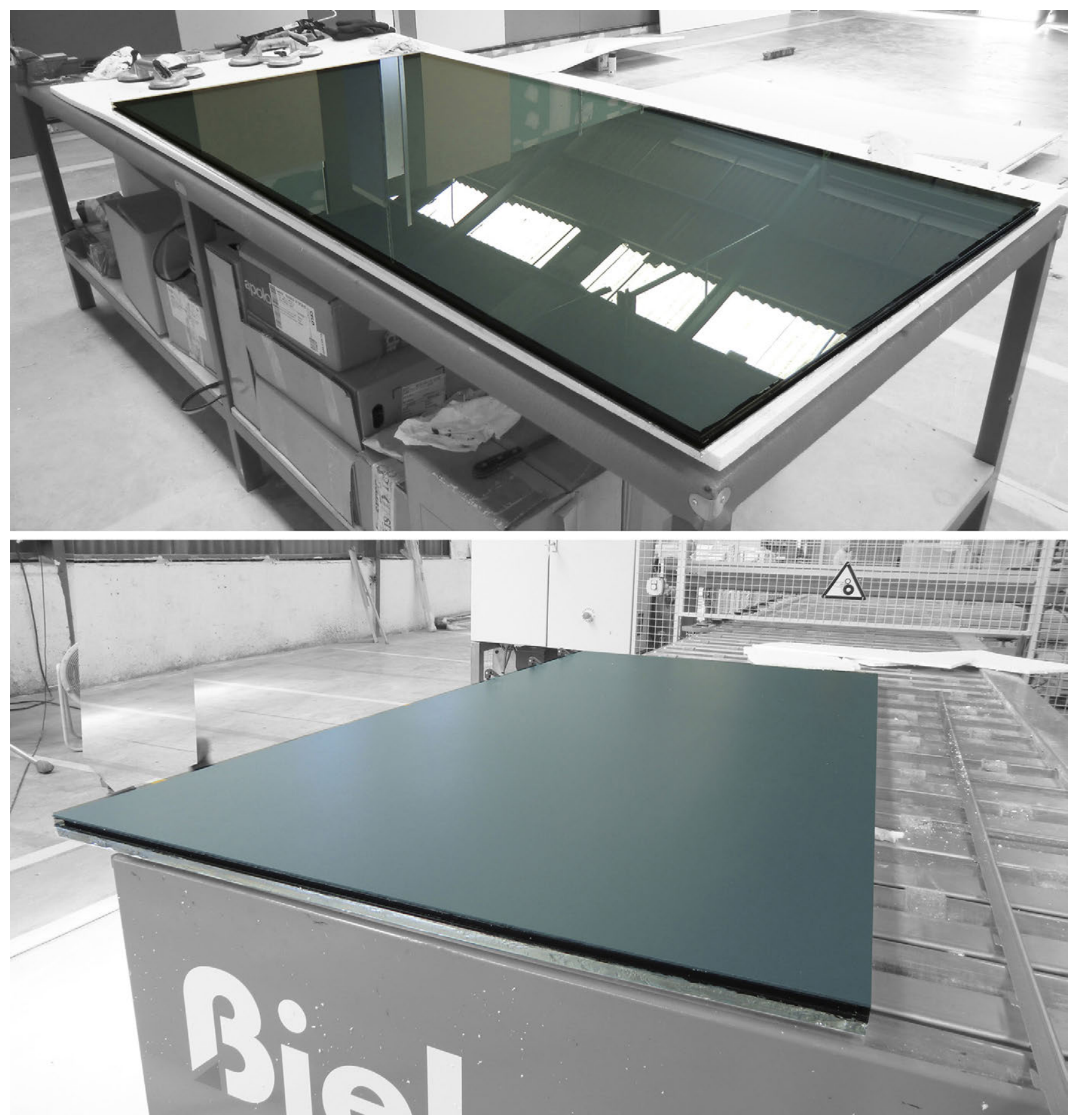

Figura 6. Prototipos de un panel cerámico colector (izquierda) y un panel cerámico no colector (derecha) (Roviras, J., 2012).

teorías analizadas (métodos multicriterio ordinales, programación matemática multiobjetivo, teoría de utilidad multiatributo, teoría de las relaciones de sobreclasificación o superación y análisis de disgregación de preferencias). Se utiliza la suma ponderada como método de agregación de preferencias, por considerarse el más adecuado entre los cinco métodos analizados dentro de la teoría de utilidad multiatributo (suma ponderada, producto ponderado, proceso analítico jerárquico, proceso analítico en red y métodos PRES).

El método de asignación de pesos utilizado es la asignación directa, por considerarse el más adecuado en este caso, entre los veinte métodos de asignación de pesos analizados (método de los pesos iguales, método de asignación directa, métodos de ordenación, métodos de comparación a partir de una sola referencia, método de comparaciones sucesivas, métodos de comparación de alternativas, métodos a partir de la diversidad en las valoraciones en cada criterio, matriz de dominación y métodos a partir de la matriz de comparación por pares). Para la selección de expertos cualificados y la realización de encuestas para la obtención de los pesos se ha seguido el método Delphi, adaptado a la ingeniería de la construcción y su gestión, tal como se muestra en Hallowell and Gambatese (10). Se ha entrevistado a un total de 13 expertos, cumpliendo todos ellos con los requisitos mínimos indicados en la anterior referencia.

Como punto de partida para la definición de los criterios, subcriterios y árbol de impacto para los sistemas de CST se ha utilizado el árbol definido en la tesis doctoral de Casanovas 

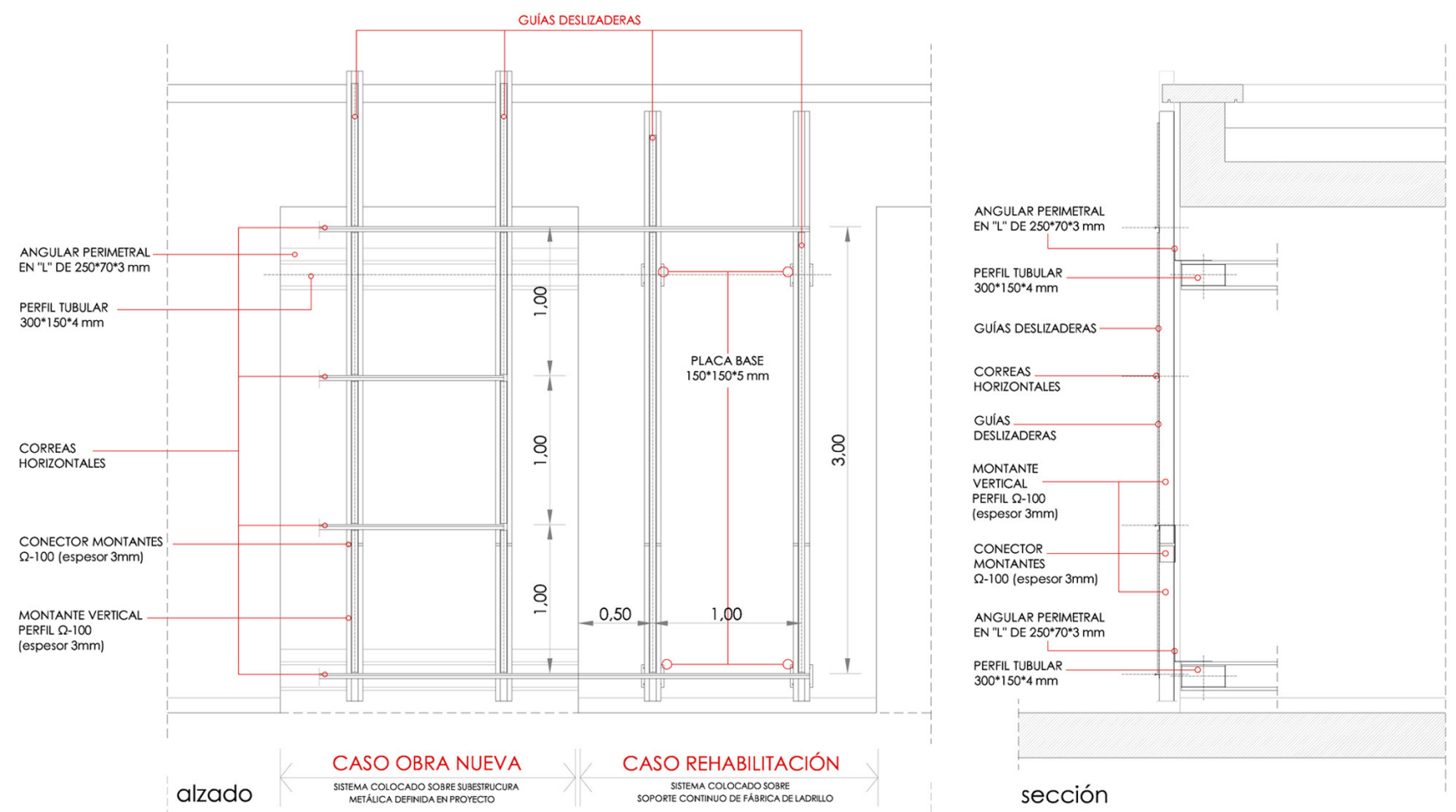

\section{DETALLE}


Figura 7. Dibujos técnicos realizados para el montaje real de fachada prototipo en las instalaciones del ITC en Castellón (Roviras, J., 2012).

(11). El objetivo del árbol allí presentado es la evaluación y priorización de procedimientos constructivos desde el punto de vista de la sostenibilidad ambiental, económica y social. Partiendo de dicho árbol se han eliminado los criterios irrelevantes para la evaluación de los sistemas de CST, se han añadido dos subcriterios que se consideran relevantes: impacto visual y ocupación de espacio y se han reordenado los criterios y subcriterios en el árbol. El árbol resultante se presenta en el siguiente apartado.

\section{5•3. Resultado}

El árbol de impacto para la evaluación y priorización de sistemas de CST resultante del proceso anterior se muestra a continuación (Figura 9). El árbol consta de tres criterios y seis subcriterios.

Para cada uno de los criterios o subcriterios se ha definido un indicador, que es la forma de medir el comportamiento de un sistema de CST y su sistema de soporte respecto al criterio o subcriterio. En la Tabla 5 se definen los indicadores de la metodología.

El cálculo del Índice de Impacto Global (IIG) se realiza mediante la ecuación [a]. El IIG es la suma ponderada de los distintos impactos considerados en la Figura 8. El impacto se define como el indicador normalizado o bien por el valor máximo entre todas las alternativas comparadas (ecuación 

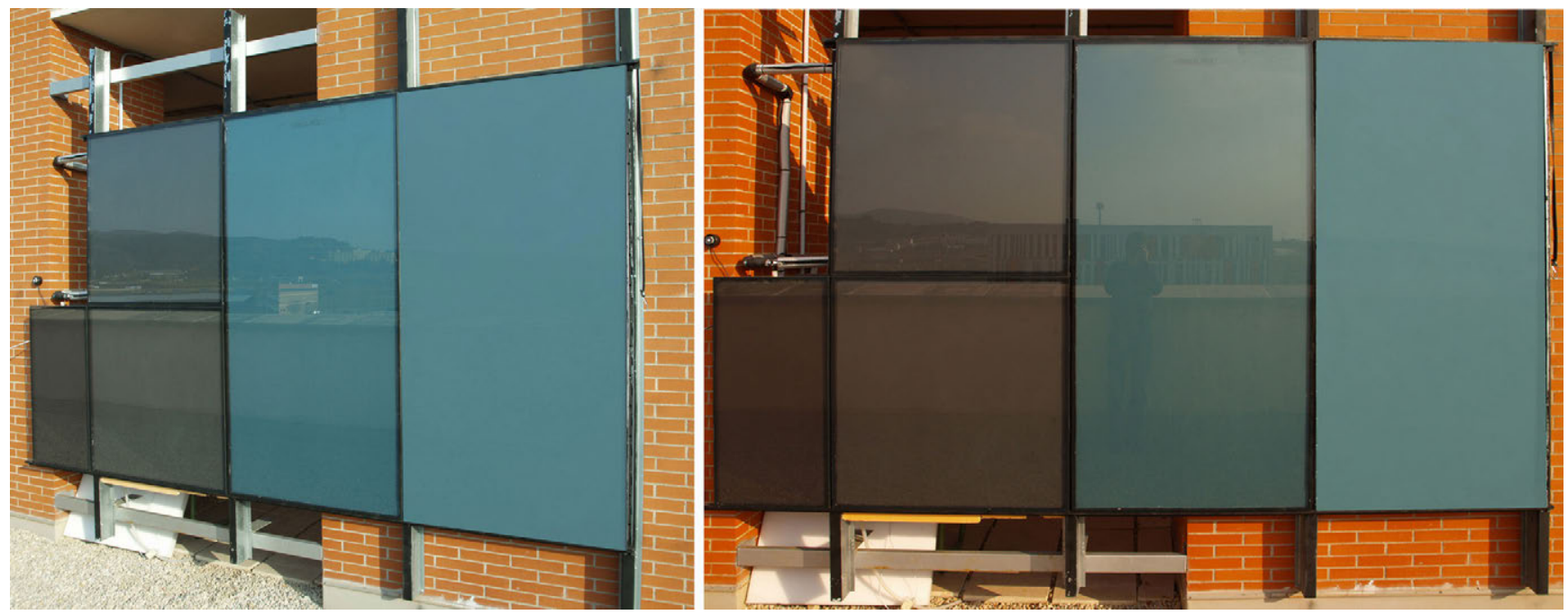

Figura 8. Prototipos construidos y activados, en fase de recogida de datos energéticos (ITC, 2013).

[b]), o bien por una alternativa tomada como referencia (ecuación [c]). Una alternativa es una combinación de sistema de CST y de soporte que se tiene en cuenta en la comparación.

$$
\begin{gathered}
\mathrm{IIG}_{\mathrm{j}}=\sum_{\mathrm{j}} \mathrm{w}_{\mathrm{j}} \cdot \operatorname{Impacto}_{\mathrm{ij}} \\
\text { Impacto }_{\mathrm{ij}}=\frac{\mathrm{I}_{\mathrm{ij}}}{\operatorname{máx}\left\{\mathrm{I}_{\mathrm{ij}}\right\}_{\mathrm{j}=\text { cte. }}} \\
\text { Impacto }_{\mathrm{ij}}=\frac{\mathrm{I}_{\mathrm{ij}}}{\mathrm{I}_{\mathrm{ref}}}
\end{gathered}
$$

Donde: i es la alternativa, $\mathrm{j}$ es el subcriterio $\mathrm{y} \mathrm{w}_{\mathrm{j}}$ es el peso global o importancia global asignado al subcriterio $\mathrm{j}$.
La Tabla 6 muestra los pesos locales y globales obtenidos como el promedio de pesos asignados por los expertos en la segunda ronda de entrevistas de acuerdo al método Delphi. Se ha llegado a un consenso entre los expertos siendo el promedio de la desviación absoluta de la mediana inferior al 10\% para el peso de todos los criterios y subcriterios.

\subsection{Conclusiones}

Se ha desarrollado una herramienta para evaluar desde una perspectiva multicriterio el nuevo colector cerámico en comparación a los colectores comerciales existentes. Como futura investigación habría que realizar simulaciones para unas necesidades de suministro de ACS en clima mediterráneo con el nuevo sistema y los sistemas comerciales existentes. A par-

\begin{tabular}{|c|c|c|}
\hline Indicador & Ud. & Comentarios \\
\hline$I_{1}=\frac{\text { Coste total }}{\text { Tiempo vida útil }}$ & $\frac{€}{\text { año }}$ & $\begin{array}{l}\text { El coste total incluye el coste de instalación, funcionamiento, mantenimiento y } \\
\text { reparación del sistema de CST y el sistema de soporte durante toda su vida útil. Hay } \\
\text { que restar el ahorro en otros materiales, en caso de que el CST sustituya elementos } \\
\text { de la cubierta y/o fachada (sistema integrado). }\end{array}$ \\
\hline $\mathrm{I}_{2}=\frac{\text { Tiempo de trabajos en altura }}{\text { Tiempo vida útil }}$ & $\frac{\mathrm{h}}{\mathrm{año}}$ & $\begin{array}{l}\text { Se contabilizan las horas de trabajos en altura (con desnivel superior a } 2 \mathrm{~m} \text { ) } \\
\text { realizados en fachadas o cubiertas inclinadas durante las operaciones de instalación, } \\
\text { mantenimiento, reparación y desmantelamiento del sistema. }\end{array}$ \\
\hline $\mathrm{I}_{3}=$ Superficie no integrada visible & $\mathrm{m}^{2}$ & Se contabiliza la superficie de CST no integrado visible desde el espacio público. \\
\hline $\mathrm{I}_{4}=$ Superficie de ocupación & $\mathrm{m}^{2}$ & $\begin{array}{l}\text { Se contabiliza la superficie en planta, tanto de espacios comunes como de espacios } \\
\text { privados, ocupada por el sistema de CST y el sistema de soporte que queda } \\
\text { inutilizada para otros usos. }\end{array}$ \\
\hline$I_{5}=\frac{\text { Peso de materiales }}{\text { Tiempo vida útil }}$ & $\frac{\mathrm{kg}}{\text { año }}$ & $\begin{array}{l}\text { Se contabiliza el consumo de materiales necesarios para la instalación, } \\
\text { mantenimiento y reparación del sistema CST y del sistema de soporte. Hay que } \\
\text { restar el peso de los materiales de fachada y/o cubierta no dispuestos debido a la } \\
\text { utilización de un sistema de CST integrado que los sustituye. }\end{array}$ \\
\hline $\mathrm{I}_{6}=\frac{\text { Energía total consumida }}{\text { Tiempo vida útil }}$ & $\frac{\mathrm{J}}{\text { año }}$ & $\begin{array}{l}\text { La energía total incluye la energía consumida en la fabricación y transporte de los } \\
\text { materiales, en la instalación, funcionamiento, mantenimiento y reparación del } \\
\text { sistema CST y del sistema de soporte durante toda su vida útil. En caso de que el } \\
\text { CST sea integrado, sustituirá a materiales de cubierta y/o fachada. Hay que restar } \\
\text { el ahorro energético en la fabricación y transporte de los materiales de cubierta y/o } \\
\text { fachada que se dejan de utilizar porque son sustituidos por el sistema de CST. }\end{array}$ \\
\hline $\mathrm{I}_{7}=\frac{\text { Emisión gases efecto invernadero }}{\text { Tiempo vida útil }}$ & $\frac{\mathrm{kgCO}_{2} \mathrm{e}}{\text { año }}$ & $\begin{array}{l}\text { Se contabilizan las emisiones producidas debidas a la fabricación y transporte de los } \\
\text { materiales, la instalación, funcionamiento, mantenimiento y reparación del sistema } \\
\text { CST y del sistema de soporte durante toda su vida útil. Hay que restar el ahorro de } \\
\text { emisiones en la fabricación y transporte de los materiales de cubierta y/o fachada } \\
\text { no utilizados porque son sustituidos por el sistema de CST en caso de que éste sea } \\
\text { integrado. }\end{array}$ \\
\hline
\end{tabular}
tir de la simulación se obtendrían los datos necesarios para

Tabla 5. Definición de indicadores (Casanovas, 2015). 
Criterios

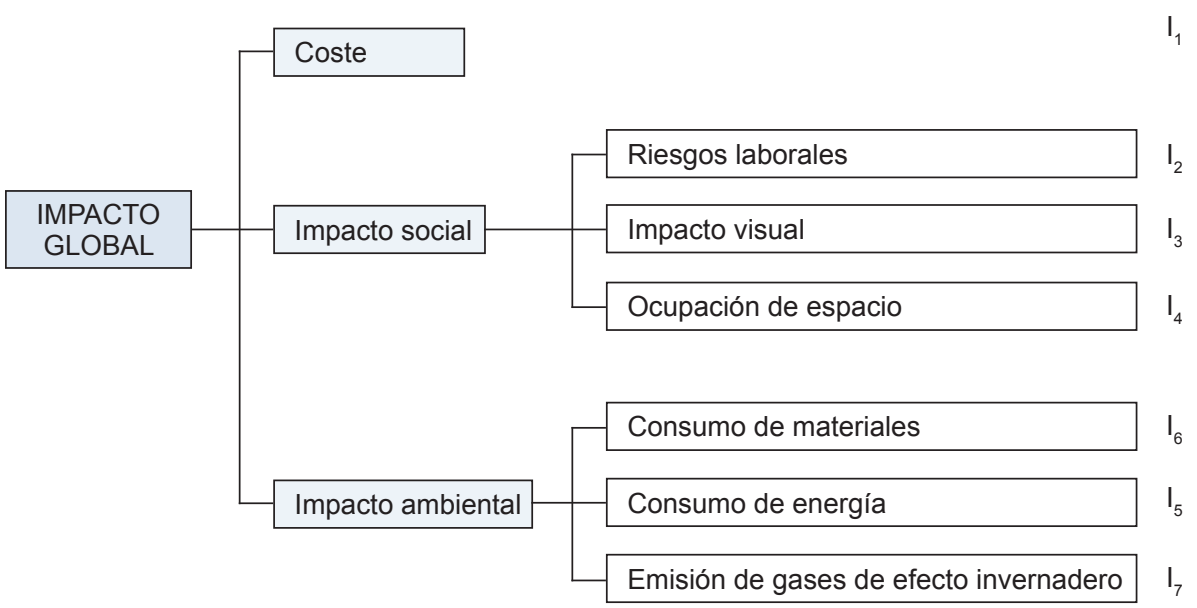

Indicadores

5

7

Figura 9. Árbol de impacto para la evaluación y priorización de los sistemas de CST (Casanovas, 2015).

poder aplicar la herramienta y calcular el IIG. Si el IIG que se obtiene del nuevo sistema cerámico se encuentra entre los IIG de los sistemas comerciales, se puede decir que el sistema cerámico, además de ser técnica, arquitectónica y energéticamente viable, bajo las condiciones de simulación para el caso estudiado, lo es desde un punto de vista multicriterio: económica, social y ambientalmente. La metodología también permite, para cualquier climatología y demanda de ACS, evaluar, comparar y priorizar entre distintos sistemas de captación solar y sistemas de soporte y elegir el que resulte más ventajoso en cada caso.

\section{CONCLUSIONES FINALES}

Se ha demostrado que es factible ejecutar una envolvente captadora mediante materiales cerámicos que cumplen las condiciones de viabilidad iniciales y que contemplan los siguientes resultados:

1. Integración arquitectónica. La envolvente captadora propuesta ofrece capacidad de juego combinatorio y variedad de acabados. La variedad de acabados que presenta la cerámica (color y textura) permite que la envolvente pueda adaptarse a cualquier requerimiento de diseño arquitectónico. El sistema diseñado permite jugar con las reflexiones y los brillos de los paneles acristalados en contraste con las superficies sin brillo de los paneles cerámicos. Por tanto se consigue que el sistema propuesto tenga suficientes recursos para integrarse arquitectónicamente.

Se ha conseguido realizar una envolvente que por un lado resuelve el cerramiento del edificio y por otro es capaz de cap- tar la energía del sol y aprovecharla para ACS y/o calefacción. Además, el anclaje de todos los paneles se realiza mediante un solo sistema constructivo, y evita el uso de subestructuras auxiliares. Por tanto, el nuevo sistema captador cerámico cumple con la doble funcionalidad constructiva y energética.

2. Beneficios económicos. Se ha conseguido desarrollar un colector solar térmico con materiales cerámicos que han sustituido a los metales y a sus respectivos recubrimientos especiales. Además, los colectores cerámicos forman parte de una envolvente captadora que sustituye al cerramiento convencional, elimina el coste añadido que generan los colectores convencionales comercializados y evita el uso de estructuras auxiliares para el anclaje de los mismos. Además, mediante la utilización de tuberías de PEX se ha conseguido evitar también el uso de elementos metálicos en el sistema de distribución y de conexionado de los paneles colectores. Por tanto, se minimiza el coste final del sistema y su respectiva amortización.

El espesor total del panel colector es de $51 \mathrm{~mm}$ (26 mm sin aislante térmico), lo que reduce aproximadamente un 30$35 \%$ el espesor de un colector plano convencional. Además, si se tiene en cuenta que este espesor forma parte de la envolvente del edificio, a diferencia de la mayoría de los comercializados que van superpuestos sobre el cerramiento, se puede concluir que el sistema aquí propuesto es altamente competitivo porque favorece una mayor superficie útil y reduce la superficie construida.

3. Sistema constructivo de la envolvente. La fachada prototipo se ha construido con operarios sin experiencia alguna en

Tabla 6. Pesos locales y globales de los criterios y subcriterios (Casanovas, 2015).

\begin{tabular}{|c|c|c|c|c|}
\hline Criterio & $\begin{array}{c}\text { Peso local del } \\
\text { criterio (\%) }\end{array}$ & Subcriterio & $\begin{array}{c}\text { Peso local del } \\
\text { subcriterio (\%) }\end{array}$ & Peso global (\%) \\
\hline Coste & 43 & - & - & 43 \\
\hline \multirow{3}{*}{ Impacto social } & \multirow{3}{*}{24} & Riesgos laborales & 38 & 9 \\
\hline & & Impacto visual & 29 & 7 \\
\hline & & Ocupación de espacio & 33 & 8 \\
\hline \multirow{3}{*}{ Impacto ambiental } & \multirow{3}{*}{33} & Consumo de materiales & 26 & 9 \\
\hline & & Consumo de energía & 43 & 14 \\
\hline & & Emisión de gases de efecto invernadero & 31 & 10 \\
\hline
\end{tabular}


la construcción de fachadas y/o cubiertas. La claridad del sistema constructivo ha permitido una correcta ejecución. Por tanto, se verifica la simplicidad de su replanteo y garantiza una construcción sencilla y segura. También se verifica en el ensayo de campo, la facilidad que ofrece el sistema de descolgar un panel sin necesidad de actuar en los paneles vecinos.

El ensayo de campo verifica el buen comportamiento estructural del sistema de anclaje propuesto, frente a las cargas permanentes generadas por el peso propio de los paneles y los perfiles metálicos y a las cargas variables producidas por el viento.

4. Adecuación al clima mediterráneo. Los ensayos en laboratorio y los ensayos de campo realizados a los distintos prototipos construidos validan los rendimientos térmicos obtenidos consiguiendo temperaturas de trabajo comprendidas entre los 30 y $45^{\circ}$. Por tanto, al considerar su aplicación en grandes superficies y en zonas con un alto índice de radiación solar, se cumple con el objetivo de diseñar una envolvente captadora cerámica con un rendimiento adecuado para el clima mediterráneo o similar.

5. Además se ha desarrollado una herramienta que permite cuantificar, evaluar y comparar el impacto global (económi- co, ambiental y social) producido por los sistemas de CST y su sistema de soporte desde un punto de vista multicriterio para todo clima y demanda de ACS. Como futuros trabajos, a partir de datos obtenidos en simulaciones, esta herramienta debe permitir evaluar el impacto del nuevo CST cerámico y compararlo con el impacto de los CST comerciales actuales. La herramienta también permite determinar las condiciones climatológicas y de demanda en las que el sistema cerámico es más adecuado dentro del clima mediterráneo.

\section{AGRADECIMIENTOS}

Los autores quieren expresar su agradecimiento al equipo de investigadores del Instituto de Tecnología Cerámica (con Vicente Cantavella al frente) y de Uralita Iberia (con Enrique Zamora al frente) que participaron en el proyecto de investigación que ha dado pie a este artículo y también al Ministerio de Industria, Turismo y Comercio por la concesión de una subvención que hizo posible la investigación en la convocatoria de ayudas del Plan Nacional de Investigación Científica, Desarrollo e Innovación Tecnológica 2008-2011.

Especial agradecimiento también a los 13 expertos que participaron en el proceso de asignación de pesos por su tiempo y por compartir sus conocimientos y opinión.

\section{REFERENCIAS}

(1) European Solar Thermal Industry Federation. (2011). Trends and Markets statistics June 2010. Solar Thermal Markets in Europe. Bruselas: ESTIF.

(2) Anderson, T.N., Duke, M., Carson, J.K. (2010). The effect of colour on the thermal performance of building integrated solar collectors. Solar Energy Materials and Solar Cells, 94(2): 350-354.

(3) Colon, C.J., Merrigan, T. (2001). Roof integrated solar absorber: The measured performance of "Invisible" solar collectors (RISA). Golden, Colorado: National Renewable Energy Laboratory (NREL).

(4) Roviras, J. (2013). Integración arquitectónica de colectores solares térmicos cerámicos para clima mediterráneo. Barcelona: Universitat Internacional de Catalunya.

(5) AENOR-CEN. (2006). UNE-EN 12975-2:2006 Sistemas solares térmicos y componentes. Captadores solares. Parte 2: Métodos de ensayo. Asociación Española de Normalización (AENOR).

(6) Ji, J., et al. (2011). Modelling and validation of a building-integrated dual-function solar collector. Proceedings of the Institution of Mechanical Engineers, Part A. Journal of Power and Energy, 225(3): 259-269. ISSN: 09576509.

(7) Juanicó, L.E. (2008). A new design of roof-integrated water solar collector for domestic heating and cooling. Solar Energy, 82(6): 481-492.

(8) Wahab, H.A., et al. (2011). Studies of control strategies for building integrated solar energy system. En Clean Energy and Technology (CET), 2011 IEEE First Conference (pp. 342-347). ISBN: 978-1-4577-1353-8.

(9) Barba-Romero, S., Pomerol, J. (1997). Decisiones multicriterio: fundamentos teóricos y utilización práctica. Alcalá de Henares: Servicio de Publicaciones de la UAH.

(10) Hallowell, M., Gambatese, J. (2010). Qualitative research: Application of the Delphi method to CEM research. Journal of Construction Engineering and Management, 136(1): 99-107.

(11) Casanovas, M. (2014). Metodología para la evaluación y seguimiento de procedimientos constructivos de forma sostenible e integrada. Barcelona: Universitat Politècnica de Catalunya BarcelonaTech. 